\title{
In vivo tracking of adipose tissue grafts with cadmium-telluride quantum dots
}

\author{
Claus J. Deglmann ${ }^{1,2}$, Katarzyna Błażków-Schmalzbauer², Sarah Moorkamp², Jens Wallmichrath², \\ Riccardo E. Giunta ${ }^{2}$, Andrey L. Rogach ${ }^{3,4}$, Ernst Wagner ${ }^{4,5}$, Ruediger G. Baumeister ${ }^{2}$, \\ Manfred Ogris ${ }^{4,5,6}$ \\ ${ }^{1}$ Centre for Hand Surgery, Microsurgery and Plastic Surgery, Schoen-Klinik Muenchen Harlaching, Munich; ${ }^{2}$ Department of \\ Hand-, Plastic- and Aesthetic Surgery, Klinikum Grosshadern, LMU Munich, Munich, Germany; ${ }^{3}$ Centre of Functional Photonics (CFP), \\ Department of Physics and Material Science, City University of Hong Kong, Hong Kong; ${ }^{4}$ Center for NanoScience (CeNS), LMU Munich, \\ Munich; ${ }^{5}$ Center for System based Drug Research, Department of Pharmacy, Pharmaceutical Biotechnolgy, LMU Munich, Munich, Germany; \\ ${ }^{6}$ Department of Clinical Pharmacy and Diagnostics, Centre of Pharmaceutical Sciences, University of Vienna, Vienna, Austria
}

Background Fat grafting, or lipofilling, represent frequent clinically used entities. The fate of these transplants is still not predictable, whereas only few animal models are available for further research. Quantum dots (QDs) are semiconductor nanocrystals which can be conveniently tracked in vivo due to photoluminescence.

Methods Fat grafts in cluster form were labeled with cadmium-telluride (CdTe)-OD 770 and transplanted subcutaneously in a murine in vivo model. Photoluminescence levels were serially followed in vivo.

Results Tracing of fat grafts was possible for 50 days with CdTe-OD 770. The remaining photoluminescence was $4.9 \% \pm 2.5 \%$ for the QDs marked fat grafts after 30 days and $4.2 \% \pm$ $1.7 \%$ after 50 days. There was no significant correlation in the relative course of the tracking signal, when vital fat transplants were compared to non-vital graft controls.

Conclusions For the first-time fat grafts were tracked in vivo with CdTe-QDs. CdTe-QDs could offer a new option for in vivo tracking of fat grafts for at least 50 days, but do not document vitality of the grafts.

Keywords Quantum dots / Adipocytes / Transplantation / Adipose tissue
Correspondence: Claus J. Deglmann Centre for Hand Surgery, Microsurgery and Plastic Surgery, Schoen-Klinik Muenchen Harlaching, Harlachinger Strasse 51, 81547 Munich, Germany Tel: +49-89-6211-2061

Fax: +49-89-6211-2062

E-mail:

cldegImann@schoen-kliniken.de

Funding by DFG Excellence Cluster Nanosystems Initiative Munich (to E. Wagner) is gratefully acknowledged.

Parts of this work were presented at the DGPRAEC (German Society of Plastic, Reconstructive and Aesthetic Surgeons) meeting on September 18, 2010, in Dresden, Germany.

Received: 16 Sep 2017 • Revised: 9 Dec 2017 - Accepted: 27 Dec 2017

pISSN: 2234-6163 • elSSN: 2234-6171 • https://doi.org/10.5999/aps.2017.01487• Arch Plast Surg 2018;45:111-117

\section{INTRODUCTION}

Fat grafting has become a widely used method for plastic surgeons and other surgical specialties in recent years [1]. Its application in aesthetic, plastic and reconstructive surgery was recently expanded by translational efforts involving adipose derived progenitor cells and their use in regenerative medicine [2]. There is increasing evidence for fat grafts to play a vital role in novel therapeutic strategies [3]. Most current research is focused on mesenchymal stem cells, while the main clinical applications still consist of fat grafts that resemble adipocyte cluster transplants, without determination or purification of single cell types. For contouring effects, many surgeons prefer the method of Coleman [1] for harvesting and reinjecting.

Still, the fate of these transplanted fat grafts in vivo remains quite undefined. The determination of graft localization in a 
transplantation target is difficult and cannot be simulated in vitro. Our objective was to create a novel model for serial fat graft in vivo tracing, without the need to harvest the graft for further investigations. Quantum dots (QDs) were evaluated in this model as a labeling agent. QDs are small semiconductor nanocrystals, with a size of usually $<10 \mathrm{~nm}$ [4]. QDs have the ability to emit fluorescence when excited with near infrared light. One of the major advantages lies in the wide absorption profile and an up to 10-fold greater molar extinction coefficient compared with organic dyes [4]. We have proven an intracellular uptake of QDs 675 in fat grafts before [5] and evaluated the use of the QDs as a new option for in vivo tracking in our current study.

\section{METHODS}

Experiments were performed according to the guiding principles for research involving animals and the German legislation on protection of animals. Approval was obtained with the local governmental animal care committee (No. 55.2-1-54-2531-5308).

BALB/c mice (Janvier, Le Genest Saint Isle, France) were housed at the Department of Pharmaceutical Biology and Biotechnology, Biotechnology and Nanomedicine, Ludwig Maximilians University, Munich. Animals were allowed to accommodate to the standard conditions of the animal facility with free access to mouse chow and water.

Subcutaneous fat was surgically harvested under sterile conditions from inguinal and abdominal region of BALB/c mouse, pooled and mechanically transected with scalpels. Collected fat grafts were diluted in $1 \mathrm{~mL} \mathrm{NaCl} 0.9 \%$ on six well plates. There was no collagenase digestion or any other further processing of the harvested fat grafts, which were used immediately for incubation and transplantation experiments. In this setting, determination of vitality was not realizable after harvesting. Fat grafts weight between 0.05 and $0.10 \mathrm{~g}$.
Cadmium-telluride QDs (CdTe-QDs) were synthesized in water according to the previously published method [6]. The negatively charged CdTe-QDs 770 had a diameter of about 6.5 $\mathrm{nm}$ and showed a characteristic near infrared excitation profile with a peak at $770 \mathrm{~nm}$ with an excitation wavelength of $740 \mathrm{~nm}$ [5].

Indocyanine green (Cardiogreen, Sigma-Aldrich, Munich, Germany) was used as a control tracing agent, because its ability to emit a fluorescence tracing signal, that can be compared in vivo to QDs in the same experimental setup.

There was no use of cell culture medium or fetal calf serum addition. For incubation experiments and short time storage saline solutions $(\mathrm{NaCl} 0.9 \%)$ were used.

For in vivo experiments with fluorescence readings, BALB/c mice could be held under general isoflurane anesthesia with a custom-built small animal anesthesia unit in the IVIS Lumina Imaging System for the recurring scanning procedure (Caliper Life Science, Hopkinton, MA, USA) (Fig. 1). Near infrared excitation was created by the IVIS Lumina Imaging System. Photoluminescence as radiance was measured in photons $/ \mathrm{s} / \mathrm{sr} / \mathrm{cm}^{2}$. Signal processing and imaging was performed with the Living Image Software 3.0 (Caliper Life Science). Received fluorescence signals were subtracted by software from the signal of the surrounding area and fused with the corresponding digital photo that was created within the IVIS Lumina Imaging System.

To determine in vivo stability of the CdTe-QDs, $0.5 \mathrm{~mL}$ of a 9 $\mathrm{nmol} / \mathrm{L}$ CdTe-QDs solution was injected subcutaneously without a fat graft in a caudocranial direction on the back of the animals under general isoflurane anaesthesia $(n=3)$. Also, indocyanine green (ICG) $(0.5 \mathrm{~mL}$ of a $4 \mathrm{nmol} / \mathrm{L}$ solution) was injected in $0.5 \mathrm{~mL}$ aliquots as a control. Photoluminescence was scanned under general anesthesia on day $0,1,2,3,4,5,6,10,16,23$, and 30 . In addition, feces and urine were collected to document excretion of the labeling substances.

To evaluate a possible correlation of the fluorescence signal Fig. 1. Experimental setup for online detection

IVIS Lumina Imaging System with custombuilt animal anesthesia unit for online fluorescence readings in living small animals under general anesthesia.
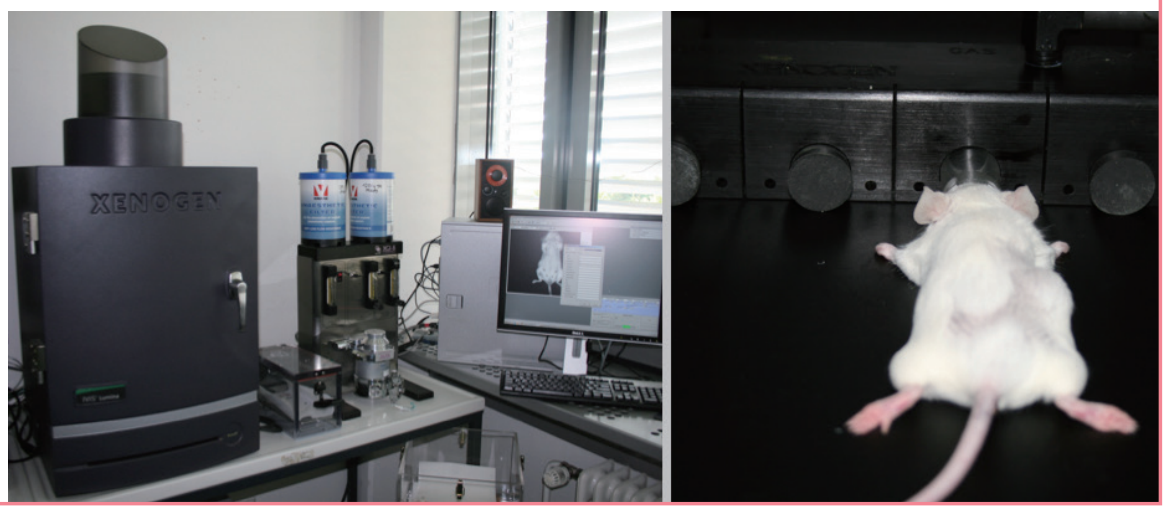
with the vitality of the fat grafts, non-vital grafts were used as control group in addition to vital fat grafts for in vivo tracking.

To create non-vital grafts as control groups, fat grafts underwent heat-treatment with $80^{\circ} \mathrm{C}$ for 45 minutes. Afterwards the grafts were cooled down to $37^{\circ} \mathrm{C}$ and incubation procedures were started. This protocol was necessary, because QDs showed strong adhesion artifacts when confronted with higher temperatures $\left(>50^{\circ} \mathrm{C}\right)$, as well as with other chemicals, like alcoholic derivatives and other chemicals that are frequently used for induction of cell death (data not shown).

For incubation experiments, harvested vital and non-vital fat grafts were incubated with $9 \mathrm{nmol} / \mathrm{L} \mathrm{CdTe}$-QDs and rinsed with $\mathrm{NaCl} 0.9 \%$ twice. After the washing procedure, the fat grafts were filled into $2 \mathrm{~mL}$ syringes and injected into the back of $\mathrm{BALB} / \mathrm{c}$ mice under general anesthesia with isoflurane and analgesia with carprofen $(n=3)$. Prior to injection experiments, the target area was shaved to avoid a reduction of photoluminescence. For injection a Strauss cannula, 15 gauge $(1.8 \times 43.0 \mathrm{~mm})$ (Dispomed Witt oHG, Gelnhausen, Germany) was used. The puncture site was sealed with 2-octyl cyanoacrylate (Dermabond, Ethicon Inc., Norderstedt, Germany). Every Mouse was scanned serially daily for the first 7 days and on day 10, 16, 23, $30,37,43$, and 50 in vivo.

\section{RESULTS}

\section{Stability of CdTe-QDs in vivo}

To examine physical stability, injected CdTe-QDs (without fat grafts) were followed in vivo, as described above. ICG was injected as a control substance.
Photoluminescence results are shown in Fig. 2. The QD 770 luminescence signal was on average reduced to $53.7 \%$ after 16 days and showed a steady decline after 4 days. ICG was on average reduced to $41.8 \%$ after 24 hours and $16.7 \%$ after 3 days. ICG showed a sharp drop until the second day and then was reduced slowly. After 30 days, a remaining luminescence of $22.6 \% \pm$ $16.5 \%$ was found for the injected QD 770 and $5.7 \% \pm 2.4 \%$ for the injected ICG.

In the ICG group, fluorescent tracer was found after 24 hours in the feces, documenting the known biliary excretion of ICG (data not shown). There was no measurable fluorescence in the ICG group urine, or in the QD 770 excrements.

\section{Fig. 2. Course of ODs injection without fat graft}

Photoluminescence time course of dorsally injected cadmium-telluride quantum dots (CdTe-QDs) 770 and indocyanine green (ICG) as control in mice. Data are normalized to the initial fluorescent values and represent (mean $\pm S D, n=3$ ).

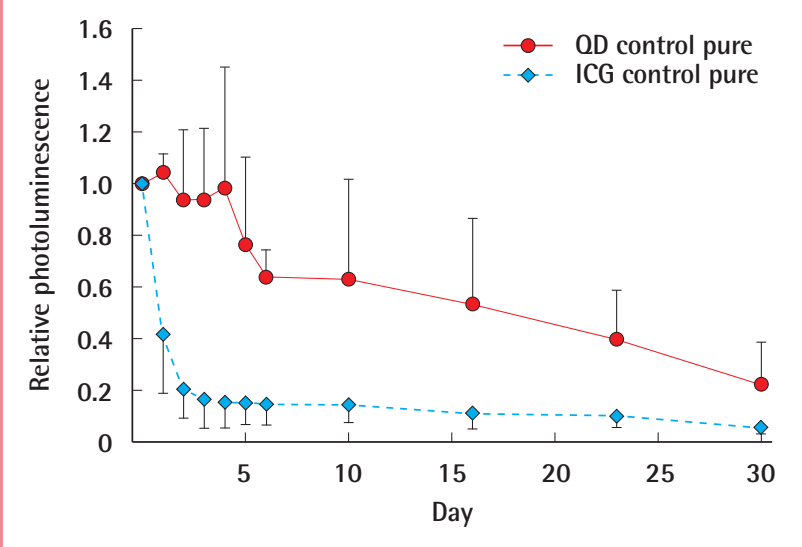

\section{Fig. 3. In vivo detection of transplanted fat clusters}

In vivo fluorescence tracing of transplanted fat clusters with cadmium-telluride quantum dots (CdTe-QDs) 770. The fluorescence reading was fused online with a picture of the target area.

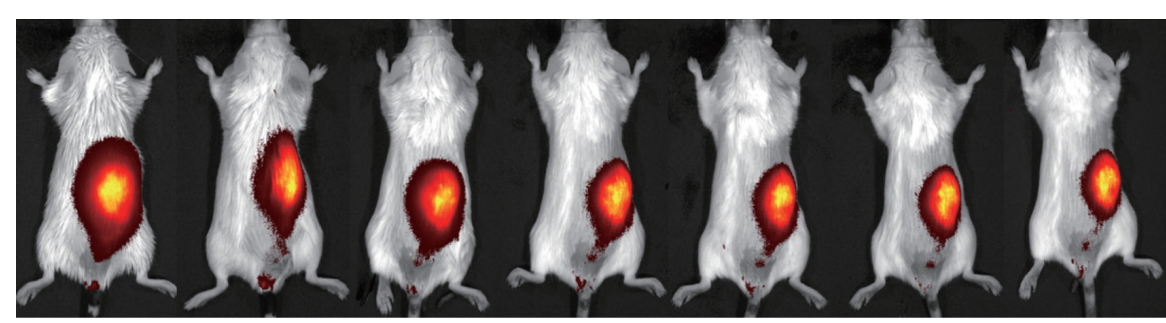

Day 0 Day 1 Day 2 Day 3 Day 4 Day 5 Day 6

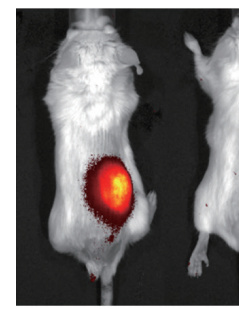

Day 10

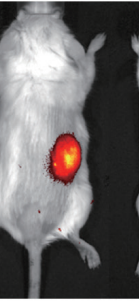

Day 16

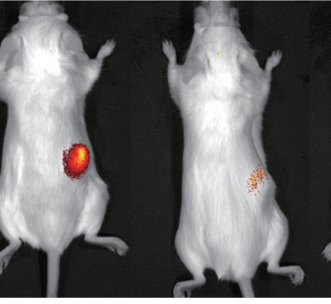

Day 23
Day 30

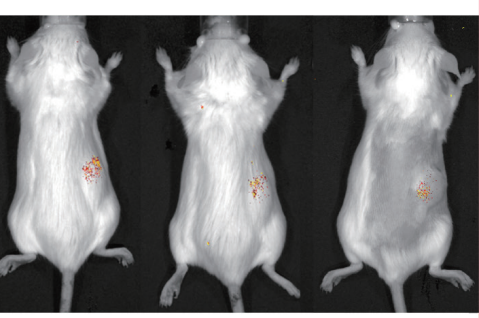

Day 37
Day 43

Day 50 


\section{Fat graft labeling with QD 770 in vivo}

Vital and non-vital fat grafts were labeled with CdTe-QD 770 and transplanted as described above.

Fluorescence signal follow-up was received from the vital and non-vital fat grafts in vivo. After 30 days the signal was deteriorated in the fusion views, but still detectable (Fig. 3).

Absolute fluorescence values (Fig. 4) showed a detectable CdTe-QD 770 signal until the 50th day in the vital fat graft group. In the non-vital group all fat grafts were detected until day 43 , whereas one graft was not detected on day 50 anymore. The average luminescence strength of the vital fat grafts was reduced more than 50\% after 72 hours. Compared to the non-vital grafts, the initial signal was 5.96 times stronger. When looked at the relative fluorescence values to even out differences in the initial fluorescence levels (luminescence/luminescence on day 0 ), we found a similar course on the first 5 days and afterwards an almost steady state in the non-vital graft group (Fig. 5).

After 30 days, the remaining fluorescent level was $4.9 \% \pm 2.5 \%$ of the initial fluorescent level in the vital fat grafts and $24.3 \% \pm$ $16.4 \%$ in the non-vital fat grafts. The non-vital fat grafts showed comparable absolute levels of photoluminescence as the vital fat grafts (Fig. 5) at this time point. At day 50, the remaining luminescence levels were $4.2 \% \pm 1.7 \%$ for the vital grafts and $6.3 \% \pm$ $5.6 \%$ with one graft not being detectable anymore from the non-vital fat grafts.

After 50 days, the tracing was terminated. Adipocyte clusters were surgically excised and openly examined under the IVIS Lumina System (Fig. 6).

\section{Fig. 4. Photoluminescence of vital and non-vital grafts}

Absolute fluorescence values (mean $\pm S D, n=3$ ) of quantum dots (QDs) 770 incubated and subcutaneous transplanted fat clusters. The control group consisted of non-vital fat grafts (NV-FG) $(n=3)$.

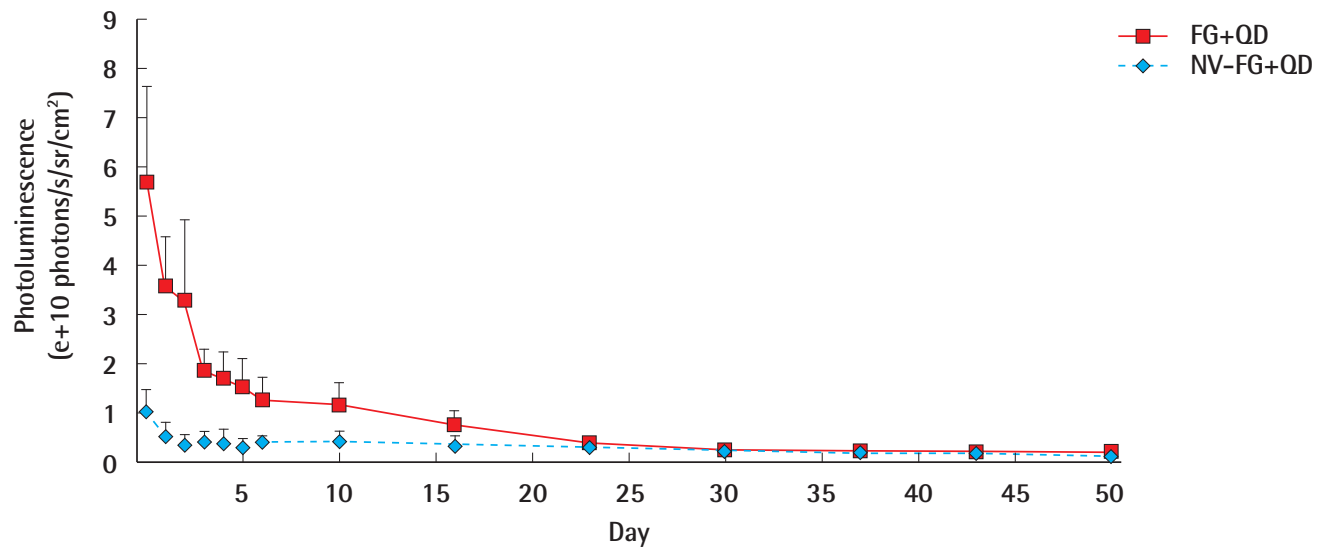

\section{Fig. 5. Normalized photoluminescence of transplanted fat grafts}

Relative photoluminescence of quantum dots (ODs) 770 (mean $\pm S D, n=3$ ) incubated and subcutaneous transplanted fat clusters, compared to the initial value. NV-FG, non-vital fat grafts.

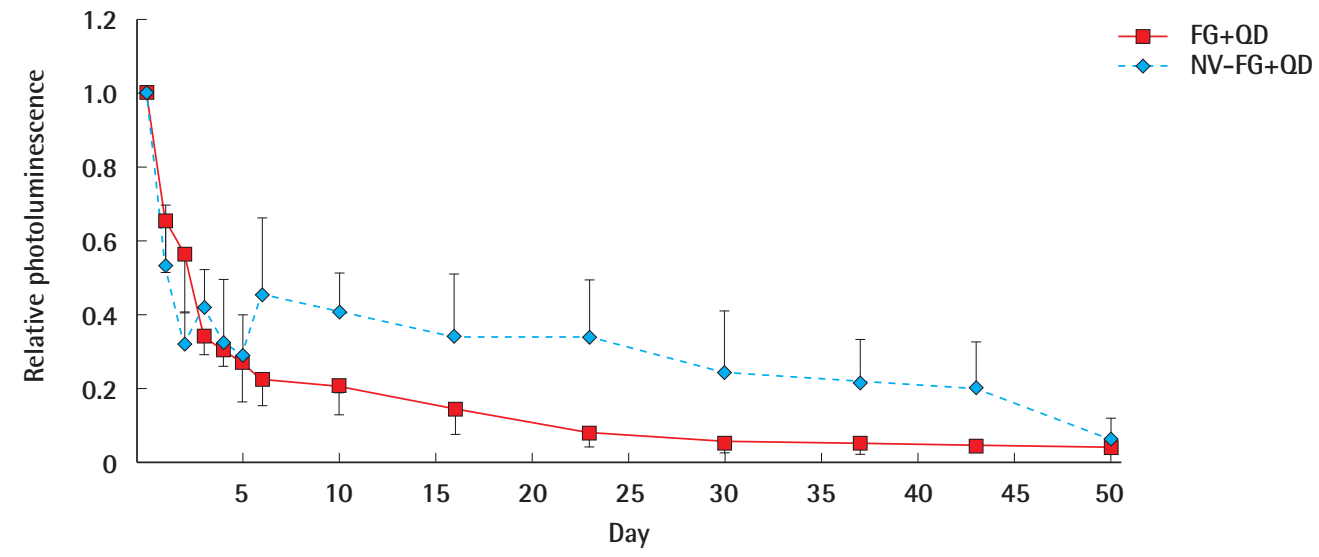




\section{Fig. 6. Examination of fat graft}

Fat cluster transplants were excised and examined 50 days after cluster labeling with cadmium-telluride quantum dots (CdTe-0D) 770: vital transplanted adipocyte clusters showed frequently increased revascularization (A). The graft result presents spherical, despite of a more areal transplantion of the fat graft, probably based on a pooling effect due to animal movement. There was a remaining direct fluorescence signal on the transplants, when examined ex vivo (B). Not all transplanted fat clusters showed an in vivo signal after 50 days.

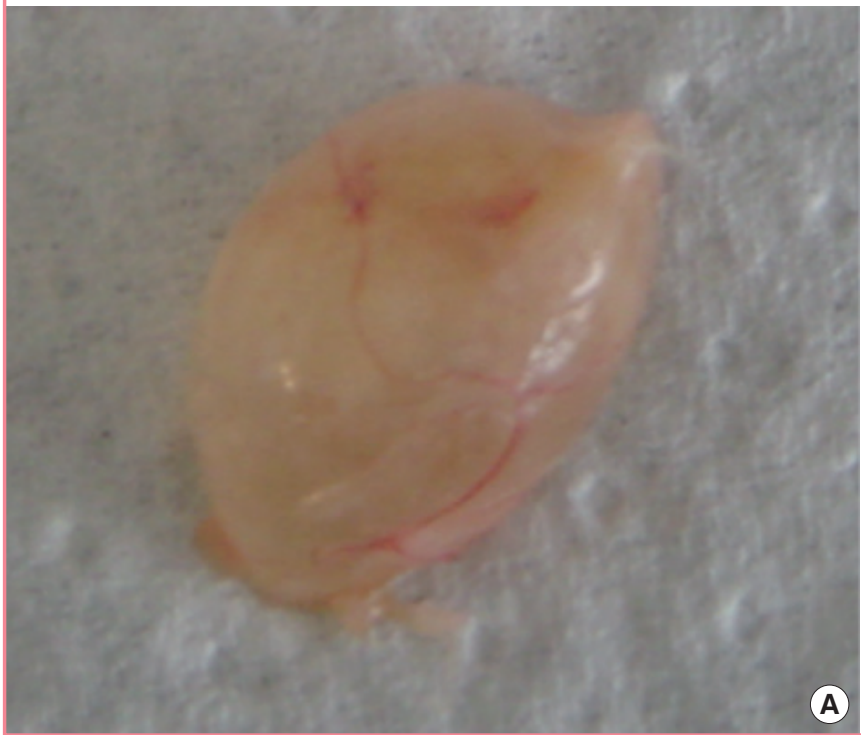

As an incidental observation, transplanted vital fat grafts showed an increased revascularization at the end of the study (Fig. 6A), if compared to transplanted non-vital fat grafts. Fat grafts showed a remaining fluorescence signal ex vivo after 50 days (Fig. 6B).

\section{DISCUSSION}

Fat cluster grafting has become a standard plastic surgical procedure $[1,7]$. Unfortunately, there is not much experimental data supporting the clinical success of the frequently employed method [8]. Partly, the lacking data is caused by a scientific focus, which is currently put mainly on progenitor or stem cells, promising a broad functionality [9].

There have been few models for the follow-up of fat derived grafts using fluorescent dye. Rieck and Schlaak [10] used PKH26, a lipophilic dye for staining a collagenized fat derived cell suspension, which was injected subcutaneous, intramuscularly and subcapsular into the kidney and was able to demonstrate a fluorescent signal even after 6 months. The actual measurement was done ex vivo in histological preparations under confocal microscopy. Also, Rieck and Schlaak [11] published a Lewis rat model for tracking of preadipocytes with PKH26 and demonstrated the examination ex vivo under confocal microscopy. Further substances for tracking of human preadipocytes were tested in vitro from Hemmrich et al. [12], stating a high toxicity for carboxyfluorescein succinimidyl ester (CFSE) and

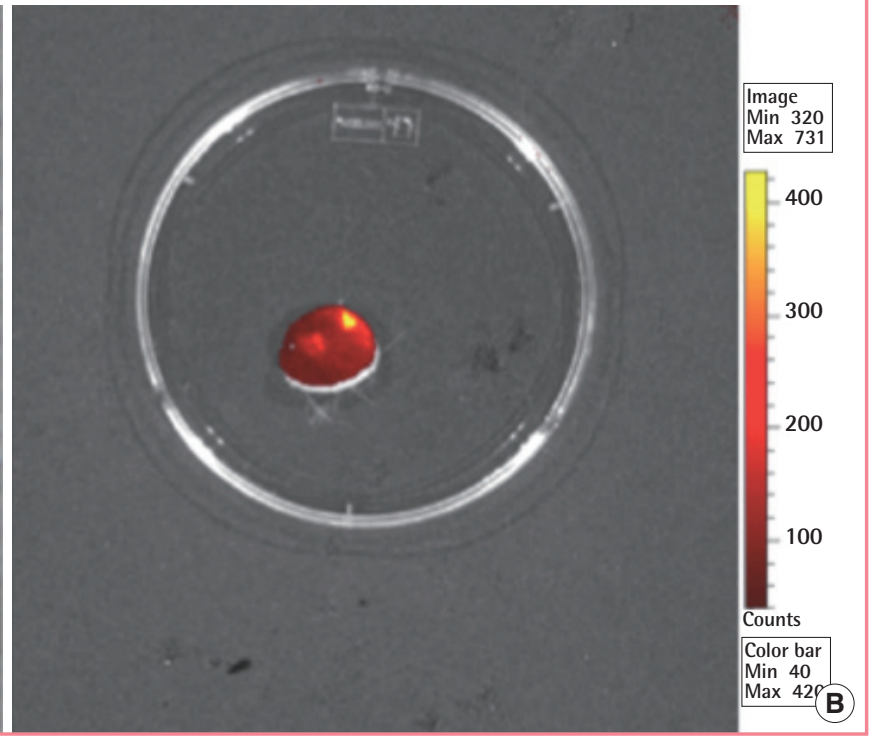

CM-DiI. CFSE, CM-DiI, and PKH 26 showed a remaining fluorescence of around $4 \%$ to $6 \%$ after 35 days in vitro. Zhou et al. [13] used luciferase transgenic rats and bioimaging to examine the difference between transplanted fat tissue with and without a stromal vascular fraction, showing a higher survival rate for the stromal vascular fraction group at 63 days. Furthermore Bliley et al. [14] used dioctadecyl-tetramethylindotricarbocyanine iodide, a near-infrared dye to track the stromal vascular fraction of fat grafts in vivo.

Our animal model clearly was inspired by the clinical use of micro fat grafts [15]. Since our model uses fat grafts in the form of micro fat cluster, there is a limitation of the scientific acuity concerning the determination of vitality, as well as cell count and origin. But on the other hand, fat alterations like collagenase digestion would have altered membrane proteins and cell viability. We were willing to take this drawback in return for an animal model that reflects the clinical reality.

QDs were introduced as nanocrystals with unique luminescent properties and stable fluorescence emission [4,16]. There has been a tremendous increase in the use of semiconductor QDs as a fluorescent label for imaging, tracing and sensing in living tissues $[5,17,18]$. There have been attempts to couple QDs to specific ligands for target specificity [17-19]. For our experiments, we used custom-built CdTe-QDs that were negatively charged and had a diameter of $7 \mathrm{~nm}$.

Yukawa et al. [20] described the use of CdSe/ZnS-core/shell nanocrystals as a potential labeling tool for adipose tissue-de- 
rived stem cells (ASCs). Also, a transfection with QDs of ASCs with a cell penetrating peptide was reported. The fluorescence intensity was maintained for at least 2 weeks with the used QDs 655 [21].

To our knowledge, our experiment presented for the first time the tracking of fat grafts in a continuous in vivo model. The chosen marker QD 770 showed the potential for a useful applicability.

The non-vital fat grafts, which were chosen as controls, showed a reduced absolute QDs uptake in our experiments, but we were not able to correlate a specific uptake to the viability of a fat graft. Therefore, the QDs labeling tool remains a tracking, rather than a viability tool, which is a limitation of the current experimental model. In vivo online tracking might play a role for follow-up of functional fat grafts for nerve cushioning, wound healing or other new indications, especially in an experimental setup. Other methods should be applied, if vitality of fat grafts is subject of an investigation.

Morphologically the fluorescent shape of the injected clusters became cohesive over time, although the fat transplantation was performed in a pearl array. This was already observed in other fat graft experiments and was earlier contributed to the movement of the animals [22]. Also, this mechanism seems to be a potential contributor for an intercalated increase of fluorescence values per area in some groups over time.

The signals also showed no hint for in vivo leaking to the surrounding of the labeling markers in the $\mathrm{QD}$ in the form of radial spreading, although leaking poses a potential problem that has to stay in the focus of further research.

QD 770 showed a sustaining labeling quality in form of a slow fading out of photoluminescence.

After 16 days in vivo tracing, there was on average $14.1 \%$ of the initial QDs fluorescence level. Therefore, QDs seem to permit tracing for medium to long-term tracking experiments.

Toxicity of QDs is to date not finally evaluated. Since the core of QDs are usually based on heavy metals, like cadmium in the used CdTe-QDs, toxicity has to be addressed in further studies [4]. We did not observe acute toxicity on microscopic evaluation. CdTe-QDs reportedly showed cytotoxic properties to a breast cancer cell line, which were not dose dependent to the intracellular Cd2+levels [23]. Other groups observed no histological changes with $\mathrm{CdSe}(0.25) \mathrm{Te}(0.75) / \mathrm{CdS}$-QDs in major organs, when administered in a mouse model, even at high doses [24]. The accumulation of nanoparticles in organs, like liver or spleen was recently described [25].

In vivo tracking of fat graft with QDs represents a new tool in the arsenal of applied nanotechnology, while further tests are necessary to exclude a potential toxicity. The application of this novel model for fat graft tracing could be useful in the evaluation of new functional and aesthetical fat graft transplants.

\section{NOTES}

\section{Conflict of interest}

No potential conflict of interest relevant to this article was reported.

\section{Ethical approval}

Approval was obtained with the local governmental animal care committee (No. 55.2-1-54-2531-53-08).

\section{REFERENCES}

1. Coleman SR. Structural fat grafting: more than a permanent filler. Plast Reconstr Surg 2006;118(3 Suppl):108S-120S.

2. Duncker DJ, Uitterdijk A, Van der Giessen WJ. Fat is not all bad: how to make good use of adipose tissue. Eur Heart J 2007;28:2565-7.

3. Sterodimas A, De Faria J, Correa WE, Pitanguy I. Tissue engineering in plastic surgery: an up-to-date review of the current literature. Ann Plast Surg 2009;62:97-103.

4. Rogach AL, Ogris M. Near-infrared-emitting semiconductor quantum dots for tumor imaging and targeting. Curr Opin Mol Ther 2010;12:331-9.

5. Deglmann CJ, Blazkow-Schmalzbauer K, Moorkamp S, et al. Cadmium telluride quantum dots as a fluorescence marker for adipose tissue grafts. Ann Plast Surg 2017;78:217-22.

6. Gaponik N, Talapin DV, Rogach AL, et al. Thiol-capping of CdTe nanocrystals: an alternative to organometallic synthetic routes. J Phys Chem B 2002;106:7177-85

7. Guerrerosantos J. Long-term outcome of autologous fat transplantation in aesthetic facial recontouring: sixteen years of experience with 1936 cases. Clin Plast Surg 2000;27:51543.

8. Bucky LP, Percec I. The science of autologous fat grafting: views on current and future approaches to neoadipogenesis. Aesthet Surg J 2008;28:313-21.

9. Yoshimura K, Suga H, Eto H. Adipose-derived stem/progenitor cells: roles in adipose tissue remodeling and potential use for soft tissue augmentation. Regen Med 2009;4: 265-73.

10. Rieck B, Schlaak S. Measurement in vivo of the survival rate in autologous adipocyte transplantation. Plast Reconstr Surg 2003;111:2315-23.

11. Rieck B, Schlaak S. In vivo tracking of rat preadipocytes after autologous transplantation. Ann Plast Surg 2003;51:294- 
300.

12. Hemmrich K, Meersch M, von Heimburg D, Pallua N. Applicability of the dyes CFSE, CM-DiI and PKH26 for tracking of human preadipocytes to evaluate adipose tissue engineering. Cells Tissues Organs 2006;184:117-27.

13. Zhou SB, Chiang CA, Xie Y, et al. In vivo bioimaging analysis of stromal vascular fraction-assisted fat grafting: the interaction and mutualism of cells and grafted fat. Transplantation 2014;98:1048-55.

14. Bliley JM, Satish L, McLaughlin MM, et al. Imaging the stromal vascular fraction during soft-tissue reconstruction. Plast Reconstr Surg 2015;136:1205-15.

15. Coleman SR. Facial recontouring with lipostructure. Clin Plast Surg 1997;24:347-67.

16. Akerman ME, Chan WC, Laakkonen P, Bhatia SN, Ruoslahti E. Nanocrystal targeting in vivo. Proc Natl Acad Sci U S A 2002;99:12617-21.

17. Zintchenko A, Susha AS, Concia M, et al. Drug nanocarriers labeled with near-infrared-emitting quantum dots (quantoplexes): imaging fast dynamics of distribution in living animals. Mol Ther 2009;17:1849-56.

18. Yong KT, Roy I, Hu R, et al. Synthesis of ternary CuInS(2)/ $\mathrm{ZnS}$ quantum dot bioconjugates and their applications for targeted cancer bioimaging. Integr Biol (Camb) 2010;2:121-
9.

19. Snyder EL, Bailey D, Shipitsin M, Polyak K, Loda M. Identification of CD44v6(+)/CD24- breast carcinoma cells in primary human tumors by quantum dot-conjugated antibodies. Lab Invest 2009;89:857-66.

20. Yukawa H, Mizufune S, Mamori C, et al. Quantum dots for labeling adipose tissue-derived stem cells. Cell Transplant 2009; 18:591-9.

21. Yukawa H, Kagami Y, Watanabe M, et al. Quantum dots labeling using octa-arginine peptides for imaging of adipose tissue-derived stem cells. Biomaterials 2010;31:4094-103.

22. Smahel J. Experimental implantation of adipose tissue fragments. Br J Plast Surg 1989;42:207-11.

23. Cho SJ, Maysinger D, Jain M, Roder B, Hackbarth S, Winnik FM. Long-term exposure to CdTe quantum dots causes functional impairments in live cells. Langmuir 2007;23:197480.

24. Yong KT, Roy I, Ding H, Bergey EJ, Prasad PN. Biocompatible near-infrared quantum dots as ultrasensitive probes for long-term in vivo imaging applications. Small 2009;5:19972004.

25. De Matteis V. Exposure to inorganic nanoparticles: routes of entry, immune response, biodistribution and in vitro/in vivo toxicity evaluation. Toxics 2017;5:29. 